\title{
Design of Visual Image System Based on Internet
}

\author{
Yufei Qian
}

Taizhou University, Taizhou, Jiangsu, China, 225300

\begin{abstract}
Key words: internet; visual image system design; requirements; influence; innovation and development
\end{abstract}

\begin{abstract}
With the progress of science and technology, Internet technology has been widely used in various fields, bringing great changes from people's work and life. At the same time, as an important part of graphic design, visual image system design is applied in various fields such as architecture, medical treatment, science and technology and media. As the main design method of corporate image and product image, under the construction of Internet, it also needs to adapt to the development of the Internet, combined with Internet technology and platform to make timely adjustments. This paper focuses on the innovation and development of visual image system design. Based on the clear design of internet technology and visual image system, this paper analyzes the requirement and influence of internet technology on the design of visual image system. Then it elaborates the visual image system design based on Internet.
\end{abstract}

\section{Internet Technology Application and Visual Image System Design}

The development of Internet technology has changed people's life and work. The convenience of the Internet for its dissemination has enabled people to get the information they need in time. It has also brought more opportunities for the widespread dissemination of visual image system design work. The Internet has enriched people's lives with the magnanimity and diversity of its information, and also brought rich reference information about the visual image system design work.

Specifically, with the development of Internet technologies and the construction and application of various Internet platforms, the combination of Internet and visual image system design is becoming more and more closely, and the two are mutually reinforcing and progress together. Spread through the Internet platform, the scope of communication has been greatly expanded, the audience widening, dissemination efficiency [1]. In addition, a variety of relying on the birth to the Internet design tools and client-side VI interface also makes the visual image system design more personalized, more in line with the needs of the audience, updated in a timely manner, the communication cycle is shortened, the economic benefits are improved. Of course, after the Internet has given the visual image system more designed platform, its own value has also been greatly exerted to promote the development of the Internet itself.

\section{Internet Technology Design Requirements for Visual Image System}

\subsection{The Diversity of Internet Technologies Requires a More Concise Visual System Design}

The development of Internet technology has developed a rich variety of design software and tools for designers to provide bright colors, lines, changing fonts and three-dimensional animation, etc, although the designer's design efficiency has indeed been improved, but rich and varied software also has higher requirements for designers. It is impossible for designers to work with these design elements to design works that meet the needs and characteristics of people. It does not mean that they can become qualified designers with a variety of design softwares. Conversely, designers must meet the audience's requirements in a colorful and graphic design effort that must be quickly and visually attractive to the audience and have the audience understand the main contents of the design in the shortest time. Therefore, when designers use Internet technology to design work, they must abide by the principle of concise design, try their best to make all kinds of elements be combined rationally, and present a clean and fresh picture so that people's attention is focused on the essentials. Including the text to be refined, the graphics should be concise, the color should be harmonious, and 
whether it is corporate image or product image are many for young audiences, so the visual image system design is best to be cool, in line with the audience's visual experience.

\subsection{The Narrow Requirements of the Internet Design Visual Image System Design and Layout more Reasonable}

Unlike the traditional design of networking to design interface, designers often have to carry out the design work within a relatively small range, especially the use of mobile terminal interfaces VI application system, the screen size is generally only $176 * 220,240 * 320$, and human limited the visual range generally focuses on the central part of the interface so that the available range of the interface can not be fully utilized. Therefore, in order to increase the limited screen available range, it is necessary to optimize the software interface design, for example, the application of 3D designs software - 3DS-MAX [2] interface designs software is a good choice. The software through the partition, the visual center as the main page, arranged around the operation keys, can perform a variety of operations, so that designers and software developers to communicate, visual image system design layout becomes more reasonable.

\section{Influence of Internet on the Design of Visual Image System}

\subsection{Visual Image System Design has Become more Sophisticated}

Under the construction of the Internet, visual image system designers can use computer graphics to show their art, not only changing the design process, but also changing the designer's design thinking, design style and design education methods, so that designers ' creativity can work well, for example, designers can use the computer graphics style and characteristics of the law to carry out creative design, which is more than the use of traditional hand-painted graphics design to better stimulate the creative potential of designers . In the traditional design of visual image system, designers must have the ability of drawing, color and fonts in graphic design, but their creativity and planning skills can not be learned in a short time. However, in the process of using computer graphics. After the design, on the one hand to promote the construction of the quality of designers, designers in a relatively short period of time to improve their modeling, color and fonts, such as painting ability and aesthetic ability, on the other hand in the design of visual image system designers based staff ability, it has also played a great role in the design process, that can help designers focuses on design creativity.

\subsection{Visual Image System Design has Become more Colourful}

The continuous development of Internet technology has made the visual image system design have more newer applications, designers can access massive amounts of useful information through the Internet, through the design work and related design knowledge around the world to learn increased knowledge, but also enriched the design theory and working model. In the actual visual image system design work, designers can use the various information on the Internet to demonstrate their own design ideas, making their own designs more creative and novelty. In addition, under the environment of Internet, the environment and the space and application of visual image system design have undergone great changes. For example, the design of visual image system has changed from traditional two-dimensional space to three-dimensional and multi-dimension, and the design of visual image system along with the development of design industry, it has also intersected with other relevant disciplines, and many design edge disciplines, interdisciplinary and emerging design disciplines have emerged. For example, the appearance of environmental labeling, product interface, image interface and new sight make the design expand from the plane to the space. The visual image system design is more three-dimensional. The dynamic visual image system design becomes the dynamic visual image system design. Multimedia designs has been promoted, web design has been developed. In short, the development of Internet technology, the construction of the Internet, making the visual image system design more richer and innovative, giving people a new design experience. 


\subsection{Visual Image System Design has Become more Scientific}

Internet technology itself is a product of technology, the Internet and visual image system design together, is bound to make visual image system design more scientific and technological. First of all, the visual image system design under the construction of the Internet has broken through the two-dimensional space design. The multi-dimensional space design makes the design work more vivid and gives each design a unique personality. Secondly, the design of the visual image system under the construction of the Internet is not a simple expression of information, but a reasonable combination of the scientific technology and the design of the visual image system, resulting in a work with scientific and technological content that is more in line with people's Logical expression, there is an invisible bridge between the audience and the work, which makes people understand the content of the information faster when they appreciate the design work. In short, the design of the visual image system under the construction of the Internet is a product of the rational combination of Internet science and technology and visual image system design. The whole process of visual image system design and the final design work are both scientific and technological.

\subsection{Visual Image System Design has Become more Ornamental}

First of all, in the Internet environment, due to the convenience and quickness of Internet information transmission, designers can understand the audience's aesthetic needs in a timely and accurate manner when designing a brand image or product image for a company, thus facilitating the designer's design work unfolding, making the design work more in line with people's aesthetic requirements. Second, the design of the visual image system under the construction of the Internet is no longer a traditional design process and form into expression. The combination of Internet technology and the visual image system design makes the design have a richer form of expression . In the past, the single visual experience was broken, the three-dimensional representation fully mobilizes all the senses and feels a visual feast, especially after the dynamic elements are added to the visual image system design, the design works will be more enjoyable .

\subsection{Visual Image System Design has Become more Spatial}

The Internet has the characteristics of not being limited by space and time . The application of Internet technology to the design of visual image system means that the design of visual image system will break with the limitation of time and space. In particular, the limitation of region will be more spatial . First of all, visual image system designs work once published on the Internet, all over the world as long as people through the Internet platform will be able to enjoy and learn the design work, making the design concept to be shared with the world, but also make the design work to maximize the value [3] . Secondly, with the development of mobile Internet technology, designers can even use the mobile Internet platform to design and produce visual image system design works, and then publish and disseminate works through the mobile internet platform, thus the design work further breaks the space limitation. In short, under the construction of the Internet, visual image system design will break through the space constraints and become more space-intensive, and will have broader space for development .

\section{Development Strategy of Internet on the Design of Visual Image System}

Make full use of Internet technology to enhance the vitality of visual image system design.The rapid development of Internet technology has changed people's life and work style , and also affected people's thinking. In the field of visual image system design, it is the working mode and design concept that affects the visual image system design. Under the construction of Internet, the design of visual image system encounters the unprecedented challenge. So we must break with the traditional design of VI and make full use of internet technology to inject fresh energy into visual image system design. Therefore, based on the characteristics of the Internet, the visual image effect is of great importance of the general public. In the Internet era, the Internet has the characteristics of free interaction and rich content. For the audience, they can browse their favorite 
content according to their own interests . For the complicated Internet information , people's visual nerve will also be greatly affected. If you want to attract the public's attention, you should make the visual design unique. For the audience, the design content of the wonderful and unique to be able to attract the public, rich design layout to highlight the diverse features . For example, with modern 3D and 4D technology, people have access to a wide range of page presentation images . During the design process, 3D or 4D stereoscopic effects can make some paintings no longer be stationary and produce a dynamic , Three-dimensional visual impact . In addition, the designers ' design methods should also be innovated with the development of science and technology, making the design work more efficient and the design works more in line with the needs of the audience . Such as to design work can be carried out on the design of the Internet, which requires designers must master a variety of innovative designs. In short, under the construction of the Internet , especially after the development of new media technologies, in order to make the visual image system design faster and more efficient, it is necessary to have sufficient Internet technology so that the visual image system design has a more convenient design method and an increased design vitality .

Make full use of Internet technology to expand the field of visual image system designs . The use of Internet technology can greatly expand the field of visual image system designs, making the design of visual image system designs work and design work through the Internet platform for production and dissemination . First of all, the application of Internet technology has changed the design method and the carrier of visual image system design . It can be designed by using Internet design tools and the Internet system to operate. The related products are designed by utilizing the dynamic and interactivity of the Internet. These are all traditional The media can not do it, it needs to design visual effects such as the effect and color of the interface, give the audience a certain visual impact, and can feel different visual experience in the visual image to attract the audience's attention, of which the color is the most attractive Eye elements in the visual image design process, we must be good at using color, from the public's visual aesthetic to seize the public eye, can have a better visual transmission effect. A variety of mobile terminal VI interfaces has also become a design carrier, is a continuation of the brand, but also makes the design work extended to more newer areas. However, the use of Internet technology to expand the visual image of the system design is also a double-edged sword, on the one hand the use of Internet-free space-time constraints make every corner of the Internet have become visual image system designs and display platform , visual image system design works. The unique charm has been greatly demonstrated, people can use computers, cell phones, etc . to appreciate and learn the design work .

On the other hand, as the field of visual image system designs and display is getting wider and wider, people also experience aesthetic fatigue . People also need a more professional and more enjoyable space for communication under the influence of mixed design work. System designs requirements are higher, so designers need to strengthen their own quality, in particular, should pay close attention to changes in the audience's aesthetic needs, timely adjustment of the design .

Make full use of Internet technology, making visual image system designed more popular and practical. Under the construction of Internet, with the further development of Internet technology, the design of visual image system will be further innovated and far-reaching . First of all, visual image system designs is almost all the basis of the design-related industries, any one related industries have to carry out visual image system design work, and visual image system designer's design thinking and philosophy will inevitably be affected by the development of Internet technology, thus affecting design related industries visual image system design . Secondly, the use of Internet technology to achieve the popularity and practicality of visual image system design , which is the inevitable development trend of visual image system design under the construction of the Internet . In order to make the visual image system design universal, in the process of visual image design, The combination of multiple visual effects to maximize the effect can be achieved through cooperation and cooperation with different Internet carriers, different carriers can play different interactive differences, and get different spreads from different feedback information systems content. For example, you can choose the combination of non-Internet carriers . Through 
an activity form, you can demonstrate different ethnic cultural information . With the active participation in the general public, there will also be a great spread effect . The premise is that visual image system design has some practicality, but to meet the practical requirements of the design work, you need to understand the market demand for visual image system designs . For example, the surging news APP uses the " Founder Yasong Song font " to highlight its elegant style, which is more attractive to users in its colorful fonts . In short, to meet the market demand, we can make visual image system design becomes more practical, and visual image system design becomes practical, natural visual image system design can be popularized .

Make full use of new media technologies to enrich the visual image system design . The combination of visual image system designs and new media technology is a fusion of design concept and information technology of science and technology. It is a multi-dimensional space expression that is closely interrelated [4]. Visual image system designs using new media technology, design and media integration, we should first use the new media technology to fully understand the characteristics of the audience, and then enrich the design of visual image system design concept, highlighting the humanistic care in the design of the idea. Secondly, the use of new media technologies and the Internet make the visual image system design break in the shackles of traditional media , more diversified in artistic expression, the expression of which is controlled by the audience's aesthetic needs, in the design process to take into account different audiences needs, try to meet the audience's visual needs . In addition, the visual image system design and the integration of new media to achieve a cross-border, or borderless design, visual image system designs to break their own traditions, the design information reorganization, although this is a visual image system design However, it also promotes the interactive design of the visual image system and embodies the characteristics of humanistic concern. As a result, the design of visual image system can not only reflect the concept of creative design but also embodies the concept of harmonious development between human and society .

\section{Conclusion}

To sum up, under the construction of the Internet, the idea of visual image system design should be explored . First, the Internet's requirements and impacts on the visual image system design should be analyzed in light of the development of Internet technology. As mentioned in the article, the visual image system design under Internet construction should More concise, the design layout should be more reasonable, the development of Internet technology will enrich the visual image system design, making the visual image system design more innovative, more scientific , appreciation and space. Then, the innovation of visual image system designs under the construction of Internet is mainly realized by actively applying various new design softwares , enriching design tools and carriers, fully understanding market demands and integrating with new media . In short, the design of the visual image system under the construction of the Internet has undergone great changes in its design process and design work. As the design of the visual image system changes, it will also affect the corporate brand image and product image associated with it .

\section{References:}

[1] Chen Jing. Internet technology under the application of print design advertising teaching reform exploration [J]. Courses Education Research, 2016 (14).

[2] Jin Yanfei talk about the combination of graphic design and technology in website construction [J]. Henan Science and Technology, 2014 (06).

[3] Cheng Xinzhu. Analysis of website design graphic design [J]. Information Technology, 2014.

[4] Wang Yinxue. Graphic design innovation under the new media [J]. Journal of Jilin Engineering and Technology Demonstration College, 2014 (05): 68-70. 